\title{
sciendo
}

\section{Radiation synthesis of poly(acrylic acid) nanogels for drug delivery applications - post-synthesis product colloidal stability}

\author{
Beata P. Rurarz ${ }^{\circ}$, \\ Natalia Gibka, \\ Małgorzata Bukowczyk, \\ Sławomir Kadłubowski, \\ Piotr Ulański $\mathbb{0}$
}

\begin{abstract}
Synthesis of polymer nanogels (NGs) for biomedical applications is considered to be a very promising application in radiation engineering. Under high-dose pulse irradiation of dilute aqueous polymer solution, reactive species generated by water radiolysis can create multiple radicals on each macromolecule and consequently induce intramolecular cross-linking of polymer chains, resulting in NG formation. The obtained products are free from harmful monomers, initiators, and cross-linking agents, which makes them potentially applicable for drug delivery applications. One of the biggest challenges in handling and use of nanoparticles, however, is the colloidal stability, when aqueous suspensions are stored for prolonged periods. Therefore, development of the best protocols for the particular nanocarrier storage is key. To address this need, we have performed the prospective study in which we systematically assessed the influence of various processing and storage scenarios feasible in our lab, on the colloidal stability of the radiation-synthesized poly(acrylic acid) (PAA) NG particles in suspension. This allowed us to choose the optimal way of handling the product after its synthesis. We confirmed that none of the strategies we used and tested are substantially detrimental to our product. Filtration with $0.2-\mu \mathrm{m}$ filters was proven sufficient for sample purification and prolonged storage in aqueous suspension did not exert a negative effect on the colloidal stability of particles suspension. We have also demonstrated that lyoprotectant-free lyophilization was suitable for our polymer nanoparticles. This is an important fact for further application of particles as nanocarriers for biologically active compounds such as targeting ligands or therapeutic moieties.
\end{abstract}

Keywords: Colloidal stability • Freeze-drying • Lyophilization • Nanogels • Poly(acrylic acid) • Radiation synthesis

B. P. Rurarz ${ }^{\bowtie}$, N. Gibka, M. Bukowczyk,

S. Kadłubowski, P. Ulański

Lodz University of Technology

Faculty of Chemistry

Institute of Applied Radiation Chemistry

Wroblewskiego 15 Str., 93-590 Lodz, Poland

E-mail: beata.rurarz@dokt.p.lodz.pl

Received: 14 October 2020

Accepted: 2 February 2021

\section{Introduction}

Nanogels (NGs) are cross-linked polymer networks that are downsized to a nanometric scale. These nanoparticles are known to have high water content, adjustable chemical and mechanical properties, and most of them are biocompatible [1]. NGs find their application in many fields, for example, in biomedicine and, especially, drug delivery systems. Due to the large surface area of NGs, various ligands can be attached to their surface [2,3]; additionally, drugs can be loaded inside the nanoparticle $[4,5]$. In light of the tremendous global need for more effective treatments with reduced adverse effects, such technological development is necessary. Currently, nanopharmaceuticals are being intensively studied and, so far, appear to be a promising advancement with respect to classical radiotherapies or chemotherapies that are commonly used in the treatment of various types of cancers [6].

The choice of proper material for a nanotherapeutic agent is of paramount importance. Application of polymers undoubtedly offers a variety of advantages which, apart from drug delivery systems, allow its

0029-5922 @ 2021 The Author(s). Published by the Institute of Nuclear Chemistry and Technology.

This is an open access article under the CC BY-NC-ND 4.0 licence (http://creativecommons.org/licences/by-nc-nd/4.0/). 
application in other medical fields such as medical diagnostics and imaging [7]. Munavirov et al. [8] claimed that poly(acrylic acid) (PAA), one of the most widely investigated biocompatible polymers, is a promising material to be used, especially in biomedicine, and its applicability is recently becoming prominent [9-13]. Attractiveness of PAA is strictly connected to the presence of carboxylic groups in the polymer structure [14]. These groups are mainly responsible for its strong association with water and sensitivity of the material to the $\mathrm{pH}$ and ionic strength changes $[8,14]$. Moreover, their presence enables convenient bioconjugation as well as interaction with other polymeric compounds, leading to formation of relatively stable polymeric nanocomplexes $[15,16]$. Although the interaction of PAA-based or PAA-coated nanoparticles with host defense systems is not yet fully understood, it is probable that such NPs would be phagocytosed by cells of the reticuloendothelial system in the liver and spleen and they can trigger the production of reactive oxygen species. The latter, in particular hydroxyl radicals, have been demonstrated to cause degradation of PAA chains into shorter fragments $[17,18]$, which is expected to facilitate their clearance from the body. Furthermore, optimization of clearance time, including the renal pathway, may be achieved in future by adjusting the NG size, cross-link density, and topology, as indicated by a study on pharmacokinetics of PAA macromolecules of various molecular weight and architecture [19]. Interestingly, it was also shown that upon interaction, PAA is able destabilize the lipid bilayers and make them more prone to leak - this effect may support the endosomal escape of the drug delivery vehicles based on PAA [8, 20-23].

Apart from the material aspect, synthetic strategy is also of great importance. It influences the safety, cost, and labour intensity connected with the carrier synthesis. With respect to poly(acrylic acid) NGs, various methods have been tested, such as emulsion polymerization from methyl acrylate in the sodium dodecylsulfate (SDS) medium [24] or precipitation polymerization of acrylic acid in water [25]. However, a method that has been explored to a great extent in recent years is radiation synthesis [26]. This procedure is simple, clean, and environmentally friendly, since the only substrates are polymer and water - no monomers, cross-linking agents, initiators, and surfactants are needed. Dilute, oxygen-free polymer solution is subjected to one or a few short, intense pulses of ionizing radiation, typically high-energy electron pulses from an accelerator. Each pulse leads to the instantaneous formation of many radicals on each polymer chain. Since the next chains are far away, these radicals recombine predominantly with each other within the single macromolecule, creating many internal, covalent cross-links. In this way, NGs are formed. By adjusting the initial molecular weight of the substrate polymer, its concentration, and irradiation parameters one can control the properties of the product. This technique, which was applied in this study, has several advantages over the traditional synthetic routes.
It involves less labour-intensive and time-consuming steps as well as safer solvents. Moreover, it offers a possibility to prepare very pure products without the need for extensive processing, lower size distribution of the NG, as well as sterilization of the product in the course of the synthesis itself [27-29]. Radiation synthesis of NGs was exercised in our research group for many years and we have shown numerous examples of radiation-induced NG synthesis from various polymers, including PAA $[27,30-36]$. The next step is to demonstrate those radiation-derived NGs applicability in the fields that can fully exploit their excellent properties.

One such important field is nanomedicine, where the superior safety and purity are key. However, in addition to all the above-mentioned important features, in nanomedicine the colloidal stability is also critical; it ensures preservation of the desired size and surface charge of the nanocarrier particles. Proper dimensions of nanostructures are vital, since very small particles $(<10 \mathrm{~nm})$ are rapidly cleared by the renal system, while on the other hand, too large structures $(>200 \mathrm{~nm})$ may lead to severe health problems (accumulation in the reticuloendothelial system, activation of the complement system, etc.) [37, 38]. Good colloidal stability ensures better efficacy and cost-effectiveness of the produced nanomedicine and therefore should be of top priority when designing the formulation. For drug delivery applications, the hydrodynamic diameter and zeta potential are the most widely used colloidal stability measures, as they allow to detect any decay, swelling/shrinking, or aggregation behaviour of the particles.

Generally, NGs are found to be rather colloidally stable structures. Ghimire et al. [39] have shown that hemoglobin-poly(acrylic acid) NG bioelectrodes can be stored at room temperature for several weeks or even steam-sterilized and do not lose their properties. Mackiewicz et al. [25] have shown that a pH-sensitive cross-linked poly(acrylic acid) NG did not exhibit much size fluctuations during 1 month of storage. Although these are relatively short periods of assessment, the results are promising and provide preliminary evidence supporting the need for further elaborate investigation, lasting up to 12 months [40]. Nevertheless, when discussing the stability of polymeric nanoparticles, one should consider not only the chemical composition of the material, but also other parameters strictly related to the particular post-synthetic fate of the product. Among others, storage temperature as well as other processes applied to improve the shelf life of the final medicine (such as freeze-drying) should be taken into consideration. Thus, especially in the case of biomedical applications, it is crucial to perform a detailed and comprehensive assessment to select the proper storage conditions that preserve the desired characteristics of the product.

Keeping in mind all the described issues, we wanted to extend our previous studies focused on the radiation synthesis of NGs, by systematically investigating the influence of various processing and storage scenarios on the size of the NG particles in suspension. This will allow us to choose the opti- 
mal way of handling the product after its synthesis, prior to further functionalization towards targeted delivery carriers of theranostic radioisotopes.

\section{Materials and methods}

\section{Materials}

Linear PAA with nominal molecular weight $\mathrm{M}_{\mathrm{w}}=450 \mathrm{kDa}$ was purchased from Polysciences Inc. (Cat. No. 03312-100, Lot: 697844; Hirschberg an der Bergstrasse, Germany) and used without further purification; concentration of the polymer is given as millimole of monomer units/ $\mathrm{dm}^{3}(\mathrm{mM})$. Perchloric acid $\left(\mathrm{HClO}_{4}, 70 \%\right)$, sodium perchlorate monohydrate $\left(\mathrm{NaClO}_{4} \cdot \mathrm{H}_{2} \mathrm{O}\right)$, and cellulose dialysis tubing of typical molecular weight cut-off $14 \mathrm{kDa}$ were purchased from Sigma-Aldrich (Poznań, Poland). $1 \mathrm{M}$ standard solution of sodium hydroxide $(\mathrm{NaOH})$ was bought from POCH (Gliwice, Poland). TKA-Micropure filtered water was used throughout the experiment. Minisart NML cellulose acetate syringe filters of the following pore sizes, $5 \mu \mathrm{m}$ (Cat. No. 17594), $1.2 \mu \mathrm{m}$ (Cat. No. 17593), $0.8 \mu \mathrm{m}$ (Cat. No. 16592), $0.45 \mu \mathrm{m}$ (Cat. No. 16555), and $0.2 \mu \mathrm{m}$ (Cat. No. 16534) were obtained from Sartorius Stedim Biotech GMBH, Göttingen, Germany.

\section{NGs synthesis}

PAA NGs were produced as previously described [36] with the modifications specified below. Briefly, an aqueous solution of PAA concentration of $17.5 \mathrm{mM}$ was prepared by overnight moderate stirring at $60^{\circ} \mathrm{C}$. On cooling, $\mathrm{pH}$ of the solution was set to 2.0 with perchloric acid (cross-linking by recombination of PAA radicals is only effective when most of the carboxylic groups are protonated). The solution was irradiated under continuous Ar-saturation, in a closed-loop system, with short pulses of electrons produced by ELU-6 linear accelerator (Eksma, Russia) with a total dose of $8.7 \mathrm{kGy}$; irradiation parameters: pulse frequency $0.5 \mathrm{~Hz}$; pulse duration $4 \mu \mathrm{s}$; electron energy $6 \mathrm{MeV}$; and dose per pulse $0.97 \mathrm{kGy}$ (determined with alanine dosimetry).

\section{NG purification and further processing}

The NG solution, obtained directly after complete irradiation, was brought to $\mathrm{pH} 7.0$ with $1 \mathrm{M}$ sodium hydroxide and equilibrated for at least $1 \mathrm{~h}$. The sample was subsequently dialyzed against water in cellulose dialysis tubing to remove the excess ions and any other low-molecular-weight impurities for seven days. After this time, the solution was filtered through a series of cellulose acetate syringe filters, down to the final filtration pore size of $0.2 \mu \mathrm{m}$. Finally, the sample was aliquoted into separate portions in conical polystyrene tubes for further processing. Each processing scenario included four major steps: freezing, storage, reconstitution, and use of varying parameters, as listed in Table 1. The freezing step was carried out in three different temperatures, either by immersion in liquid nitrogen or by loading samples onto the precooled freezer. Next, the samples were subjected to storage for particular periods: half the samples were freeze-dried and the lyophilized cakes were kept at $4^{\circ} \mathrm{C}$, while the remaining samples were stored under refrigeration until reconstitution. Upon reconstitution - either by redispersion in water or thawing, the samples were used for dynamic light scattering (DLS) measurements for up to a month and for the whole period of use they were kept at $4^{\circ} \mathrm{C}$. Detailed descriptions of the particular processing steps are provided in Table 2. The control sample was refrigerated for the whole duration of the experiment and measured as specified in the sample use period part.

\section{NG characterization}

To confirm the formation of NGs upon irradiation, weight-average molecular weight and radius of gyration (static light scattering - SLS) as well as the hydrodynamic radius (dynamic light scattering - DLS) of the obtained polymer structures were determined using BI-200SM goniometer (Brookhaven Instruments Corporation) with an Innova $90 \mathrm{C}$ Ar ion laser $(\lambda=514.5 \mathrm{~nm})$ at $25.0 \pm 0.1^{\circ} \mathrm{C}$. The sample of NG solution (without further purification and processing) was supplemented with $\mathrm{NaClO}_{4}$ to a final concentration of $0.5 \mathrm{M}$ and the $\mathrm{pH}$ was set to 10.0 with $1 \mathrm{M}$ sodium hydroxide to ensure

Table 1. Details on the samples' possible processing scenarios

Processing, storage, and sample usage parameters

Freezing conditions

Reconstitution strategy

Stock storage period

Sample use period (after stock reconstitution)

\section{Options}

Flash freezing (liquid nitrogen, $-196^{\circ} \mathrm{C}$ )

Fast freezing (precooled freezer, $-70^{\circ} \mathrm{C}$ )

Slow freezing (precooled freezer, $-22^{\circ} \mathrm{C}$ )

Freeze-drying and redispersion

Thawing

Immediate reconstitution

1-week storage

1-month storage

Immediate measurement

Measurement after 1 week

Measurement after 1 month 
Table 2. Detailed description of various processing steps applied to the samples

\begin{tabular}{ll}
\hline Flash freezing & Aliquoted samples were immersed in liquid nitrogen for at least 3 min, until visual inspection of \\
& samples confirmed full freeze. For prolonged storage, stock samples were moved to the precooled \\
& freezer $\left(-70^{\circ} \mathrm{C}\right)$.
\end{tabular}

Fast freezing Aliquoted samples were placed in $-70^{\circ} \mathrm{C}$ precooled freezer for at least $12 \mathrm{~h}$. For prolonged storage, stock samples were kept in the $-70^{\circ} \mathrm{C}$ precooled freezer.

Slow freezing Aliquoted samples were placed in $-22^{\circ} \mathrm{C}$ precooled freezer for at least $12 \mathrm{~h}$. For prolonged storage, stock samples were kept in $-22^{\circ} \mathrm{C}$ precooled freezer.

Thawing Reconstitution was realized by placing the stock sample at $4^{\circ} \mathrm{C}$ for thawing. The reconstituted sample was stored at $4^{\circ} \mathrm{C}$ for the whole sample use period.

Freeze-drying All stock samples, frozen in different conditions, after overnight treatment, were placed together and redispersion in precooled freeze-dryer, at high vacuum, with a condenser surface temperature of $-50^{\circ} \mathrm{C}$, for $120 \mathrm{~h}$. Stock samples in the form of freeze-dried cakes in conical tubes were vacuum-sealed and stored at $4^{\circ} \mathrm{C}$ until reconstitution. Redispersion was realized by adding $10 \mathrm{~mL}$ of ultrapure water to the cake and overnight moderate stirring at $37^{\circ} \mathrm{C}$. Redispersed sample was stored at $4^{\circ} \mathrm{C}$ for the whole sample use period.

Stock storage Is considered to start when the processing of the particulate sample is completed, e.g., in the period case of freeze-dried samples for redispersion - after completed lyophilization, and in the case of frozen samples for thawing - after samples are placed in the freezer.

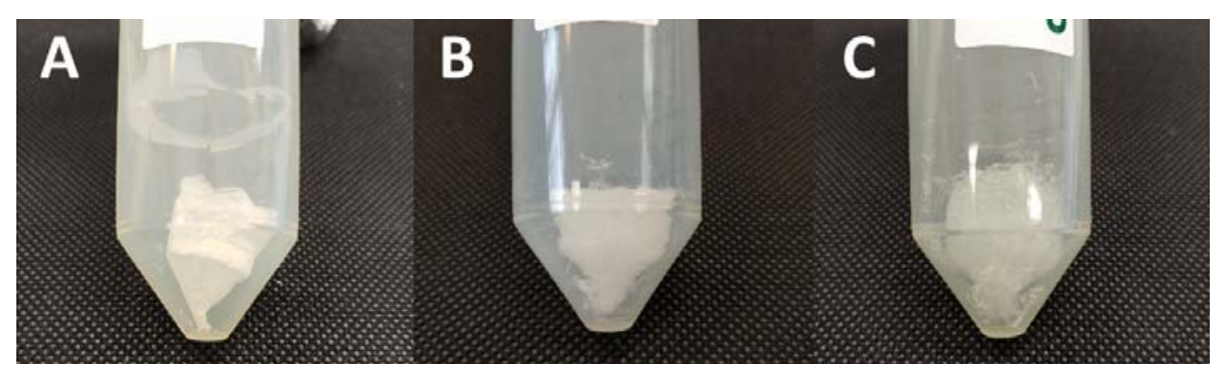

Fig. 1. Freeze-dried cakes after completed lyophilization: (A) flash freezing; (B) fast freezing; (C) slow freezing.

compact conformation of macromolecules needed for the described light scattering measurement. SLS data was processed using the Zimm algorithm, with $d n / d c=0.30 \mathrm{~cm}^{3} \cdot \mathrm{g}^{-1}$ [17]. Prior to measurement, each sample was filtered through a filter of $0.45 \mu \mathrm{m}$ pore size to remove the potential dust particles and the solvent $\left(0.5 \mathrm{M} \mathrm{NaClO}_{4}\right.$ in water, $\left.\mathrm{pH} 10.0\right)$ was filtered through a filter of $0.2 \mu \mathrm{m}$ pore size.

The size of the purified and processed NGs, expressed as a mean hydrodynamic diameter $\left(D_{h}\right)$, was measured upon reconstitution using DLS with ZetaSizer Nano ZS (Malvern) with built-in He-Ne laser $(\lambda=633 \mathrm{~nm})$ at $22.0 \pm 0.1^{\circ} \mathrm{C}$, without any additional supplementation. The $\mathrm{pH}$ of the reconstituted solutions was between 7.0 and 8.0. Prior to each measurement, the sample portions were filtered through a filter of $0.45 \mu \mathrm{m}$ pore size to remove the potential dust particles. The particle size reported in this work is the average of five measurements for each sample consisting of the automatically determined number of consecutive runs.

\section{Results}

\section{NG formation}

Radiation synthesis of NGs with a dose of $8.7 \mathrm{kGy}$ in a closed-loop system yielded moderately dense polymer structures with a weight-average molecular weight of $1650 \pm 17 \mathrm{kDa}, R_{g}$ of $48 \pm 4 \mathrm{~nm}$, and $R_{h}$ of $35 \pm 1 \mathrm{~nm}$ (measured in $0.5 \mathrm{M} \mathrm{NaClO}_{4}, \mathrm{pH} \mathrm{10}$ ), resulting in an $R_{g} / R_{h}$ ratio of 1.4 . In comparison, the $R_{g} / R_{h}$ ratio obtained for linear PAA used in this experiment was typically around 2.9 ; this substantial decrease confirms the successful cross-linking of the chains and NG formation [41].

\section{NG freeze-drying}

Although all the cakes obtained by freeze-drying of the purified NG solution were heavily collapsed (Fig. 1), there were no problems with sample redispersion. One can, however, notice the difference in the appearance of the freeze-dried solids between samples frozen at different temperatures. Sample A, flash frozen with liquid nitrogen, is compact and opaque, while on the contrary, sample $\mathrm{C}$, frozen in a precooled freezer at $-22^{\circ} \mathrm{C}$, resembles a light, fluffy mesh. Sample B, frozen at $-70^{\circ} \mathrm{C}$ represents the intermediate state - the mesh is much denser than in the case of $\mathrm{C}$, but not as compact as in the case of $\mathrm{A}$.

\section{Influence of the reconstitution strategy}

Particles were characterized immediately after synthesis and further purification and processing, in order to investigate the influence of the reconstitution strategy on the size of the product 


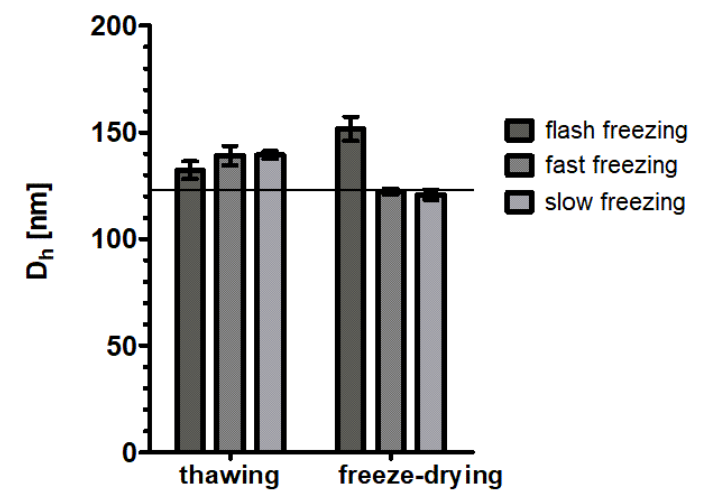

Fig. 2. Influence of the processing method on the mean particle size. Data for samples frozen using different options indicated in the graph, and, directly afterwards, subsequently subjected either to thawing or freeze-drying followed by immediate redispersion in water. The straight line indicates the mean size of the control aliquot of NGs. Error bars indicate standard deviations of mean.

(Fig. 2). Control nanoparticles, which were not further processed after purification, were of size $123 \pm 2 \mathrm{~nm}$. After reconstitution, it can be seen that there is no substantial change in the particle size, except for freeze-dried and redispersed ("freeze-drying") sample frozen in liquid nitrogen ("flash freezing"), in the case of which the size of particles was $152 \pm 6 \mathrm{~nm}$.

\section{Influence of the stock storage time}

It was also evaluated how the time of the stock samples storage in the frozen or freeze-dried state influenced the size of reconstituted nanoparticles (Fig. 3). It can be noticed that in general there are no adverse effects on the particles size on storage for most of the investigated conditions. However, for the samples stored as slow frozen stock for later thawing, there is some change in the size of nanoparticles. Considering the problems with sample filtering (high pressure required to push the sample through the filter), which occurred in some of those samples, there might be some abnormalities, which need further investigation.

\section{Influence of the time of use}

After reconstitution of the stored stocks, the nanoparticles in aqueous solution are supposed to be suitable for further modifications and in-vitro experiments for up to 1 month. Therefore, to evaluate

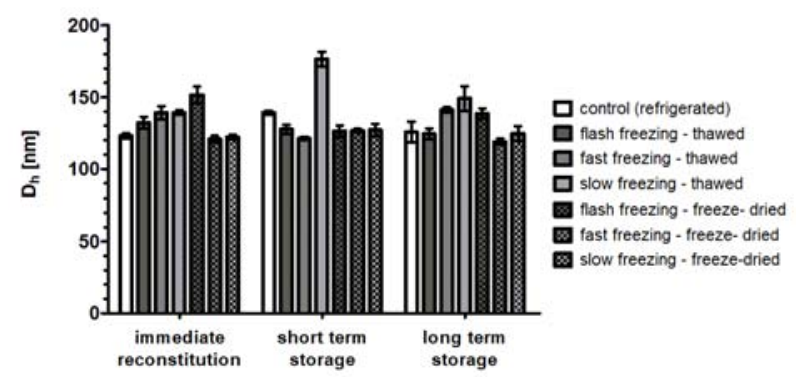

Fig. 3. Influence of the stock storage time on the mean particle size. Error bars indicate standard deviations of mean.
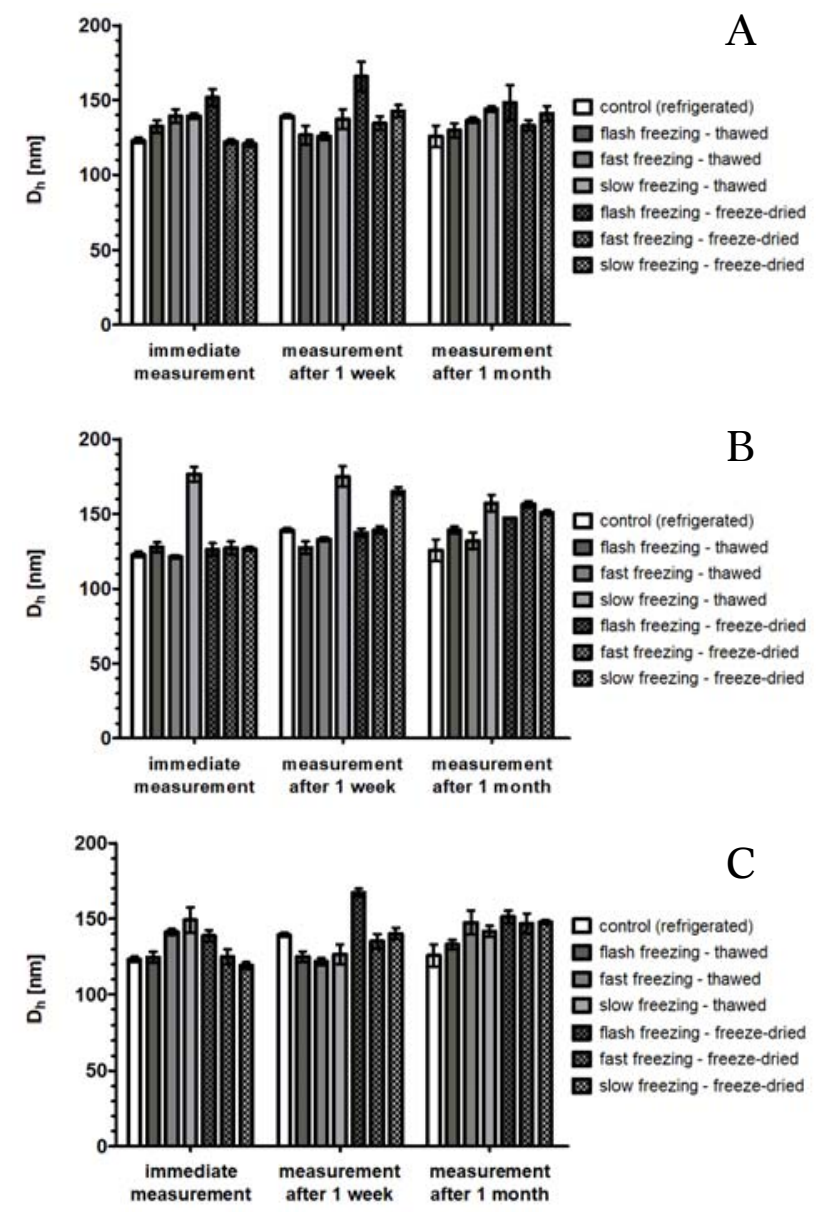

Fig. 4. Influence of the storage time on the mean particle size during prolonged use: (A) immediate reconstitution; (B) short-term storage (1 week); (C) long-term storage (1 month). Error bars indicate standard deviations of mean.

the shelf life of the reconstituted nanoparticles, we examined the stability of the nanoparticles during 1-month usage, considering the stock storage scenario. The results are shown in Fig. 4. In general, despite a slight increase, samples maintain the nanometer size, even if used for 1 month. Yet, it can be seen that some samples show a more prominent increase in size: nanoparticles that are slowly frozen and briefly stored in the freezer for subsequent thawing grew in size; however, this effect did not manifest itself so noticeably in the other storage periods. Such frozen samples also seem to produce higher $D_{h}$ results when used for a longer period. Flash-frozen freeze-dried samples seem to express some changes in size when immediately reconstituted or stored for a longer period.

\section{Influence of the complete processing scenario}

Figure 5 shows a representative comparison of the nanoparticle size when different scenarios are applied to the particles handling for a period of 1 month: doing nothing (1-month control), freezing for immediate thawing (1-month use), or storing in the freezer for later thawing (1-month storage). It can be seen, as shown previously, that in general, samples retain the nanometer size; however, there 


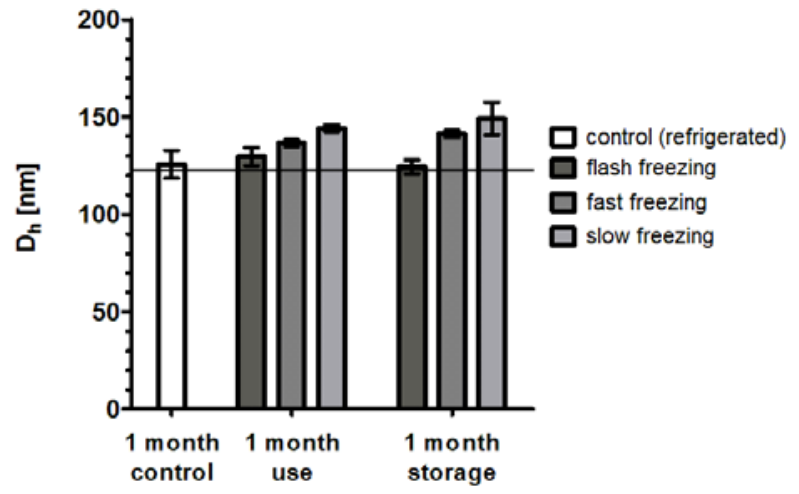

Fig. 5. Influence of the treatment scenario on the mean particle size 1 month after completed synthesis and purification. Error bars indicate standard deviations of mean.

is some influence of the freezing temperature - the higher the temperature of freezing, the more prominent the size change, as shown in Fig. 5.

\section{Discussion}

Radiation-derived products such as polymer NGs can be obtained in safe, environmentally friendly processes at favourable costs and effort. It makes them perfectly suitable for applications in cutting-edge fields, such as nanomedicine, where purity and cost-effectiveness are essential. However, next to these important features, others, such as colloidal stability, are critical and need to be carefully investigated with respect to the consecutive processes applied to the samples. In our ongoing research on the radiation synthesis of polymer NGs, we experienced some issues regarding the colloidal stability, and so far, it was approached by trial-and-error attempts. Therefore, we have performed a preliminary study in which the influence of various processing and storage scenarios on the colloidal stability of the radiation-synthesized NG particles in suspension was systematically assessed. So the gained knowledge should help to choose the optimal way of handling the product after its synthesis.

In general, it has been confirmed that none of the strategies we employed in our lab and tested were substantially detrimental to our product. The very encouraging outcome of the conducted study is also the fact that the control sample, stored at $4^{\circ} \mathrm{C}$ for the whole duration of the experiment, does not show any signs of decay, despite no special measures being applied for its preservation. Filtration with $0.2 \mu \mathrm{m}$ filters seems to be sufficient for sample securing, and prolonged storage in aqueous suspension does not exert a negative effect on the stability of particles in suspension. Such a storage scenario appears to be very convenient when the sample is used by a team in a lab. Yet, if the sample is intended to be shipped from one research laboratory to another - which is the case for our future studies, lyophilization comes in handy as a first-choice preservation procedure. We have demonstrated that this procedure is suitable for our PAA nanoparticles, despite some earlier doubts. All the samples were easily redispersible and reconstituted NGs did not show any pronounced deviations in stability throughout the experiment. This is a very important fact for further application of particles as nanocarriers - if any stability problems occur following NG conjugation with targeting ligand or therapeutic moieties, it can be concluded that these issues are not based on the inherent properties of the polymer nanocarrier.

After careful analysis, we finally conclude that the optimal way of nanoparticle handling is to use fast freezing and subsequently freeze-drying. Fast freezing seems to represent some kind of optimum freezing conditions with respect to all other factors influencing the nanoparticles in the presented experiment. Samples treated in this way exhibit the most consistent behaviour throughout the study, among all the examined scenarios.

For samples frozen with liquid nitrogen, it can be seen in Fig. 1A that a very compact structure of cake was obtained, which is a consequence of small pores resulting from small ice crystals formed during flash freezing. These small pores can be the reason for hindered water vapour escape during primary drying in the freeze-drying process and the resultant relatively high residual moisture in the freeze-dried cakes, especially if secondary drying is omitted. One can also see that the flash-frozen sample is the most heavily collapsed. All these factors are interlinked and can lead to reconstitution and stability issues in longer time periods. On the other hand, as slow freezing is applied to the sample (as shown in Fig. 1C), growing ice crystals and hence subsequent pores, are much larger and the obtained cake is more meshed. This, in turn, may lead to greater mechanical stress exerted on nanoparticles, leading to their destabilization or even fusion [42]. Another way of solving this issue may be the use of cryoprotectants, such as, very popular in this application, sugars (trehalose, sucrose, glucose [43]). Immersion of nanoparticles in the glassy matrix of applied cryoprotectant can protect them against mechanical stress exerted by growing ice crystals and hence impede their aggregation or fusion.

Acknowledgments. This work has been performed in the framework of the research project UMO2019/33/B/ST5/02125 financed by the National Science Centre, Poland. This work was supported by the National Center of Research and Development (Warsaw, Poland) in the framework of the project InterChemMed (POWR.03.02.00-00-I029/16), cofunded by the European Social Fund.

\section{ORCID}

B. P. Rurarz (D) http://orcid.org/0000-0001-6635-9508 P. Ulański (D) http://orcid.org/0000-0002-4310-3574

\section{References}

1. Oh, J. K., Lee, D. I., \& Park, J. M. (2009). Biopolymerbased microgels/nanogels for drug delivery applica- 
tions. Prog. Polym. Sci., 34(12), 1261-1282. DOI: 10.1016/j.progpolymsci.2009.08.001.

2. Dispenza, C., Adamo, G., Sabatino, M. A., Grimaldi, N., Bulone, D., Bondì, M. L., Rigogliuso, S., \& Ghersi, G. (2014). Oligonucleotides-decorated-poly(N-vinyl pyrrolidone) nanogels for gene delivery. J. Appl. Polym. Sci., 131(2), 1-8. DOI: 10.1002/app.39774C.

3. Picone, P., Sabatino, M. A., Ditta, L. A., Amato, A., San Biagio, P. L., Mulè, F., Giacomazza, D., Dispenza, C., \& Di Carlo, M. (2018). Nose-to-brain delivery of insulin enhanced by a nanogel carrier. $J$. Control. Release, 270, 23-36. DOI: 10.1016/j.jconrel.2017.11.040.

4. Argentiere, S., Blasi, L., Ciccarella, G., Barbarella, G., Cingolani, R., \& Gigli, G. (2009). Synthesis of poly (acrylic acid) nanogels and application in loading and release of an oligothiophene fluorophore and its bovine serum albumin conjugate. Macromol. Symp., 281(1), 69-76. DOI: 10.1002/masy.200950709.

5. Argentiere, S., Blasi, L., Morello, G., \& Gigli, G. (2011). A novel pH-responsive nanogel for the controlled uptake and release of hydrophobic and cationic solutes. J. Phys. Chem. C, 115(33), 16347-16353. DOI: 10.1021/jp204954a.

6. De Jong, W. H., \& Borm, P. J. (2008). Drug delivery and nanoparticles: applications and hazards. Int. J. Nanomed., 3(2), 133-149. DOI: 10.2147/ijn.s596.

7. Molina, M., Asadian-Birjand, M., Balach, J., Bergueiro, J., Miceli, E., \& Calderón, M. (2015). Stimuli-responsive nanogel composites and their application in nanomedicine. Chem. Soc. Rev., 44(17), 6161-6186. DOI: 10.1039/c5cs00199d.

8. Munavirov, B. V., Filippov, A. V., Rudakova, M. A., \& Antzutkin, O. N. (2014). Polyacrylic acid modifies local and lateral mobilities in lipid membranes. J. Dispers. Sci. Technol., 35(6), 848-858. DOI: 10.1080/01932691.2013.823096.

9. Miao, X., Ho, S. L., Tegafaw, T., Cha, H., Chang, Y., Oh, I. T., Yasenn, A. M., Marasini, S., Ghazanfari, A., Yue, H., Chae, K. S., \& Lee, G. H. (2018). Stable and non-toxic ultrasmall gadolinium oxide nanoparticle colloids (coating material = polyacrylic acid) as high-performance: T1 magnetic resonance imaging contrast agents. RSC Adv., 8(6), 3189-3197. DOI: 10.1039/c7ra11830a.

10. González-Gómez, M. A., Belderbos, S., Yañez-Vilar, S., Piñeiro, Y., Cleeren, F., Bormans, G., Deroose, C. M., Gsell, W., Himmelreich, U., \& Rivas, J. (2019). Development of superparamagnetic nanoparticles coated with polyacrylic acid and aluminum hydroxide as an efficient contrast agent for multimodal imaging. Nanomaterials, 9(11), 1-20. DOI: 10.3390/ nano9111626.

11. Khandhar, A. P., Liang, H., Simpson, A. C., Reed, S. G., Carter, D., Fox, C. B., \& Orr, M. T. (2020). Physicochemical structure of a polyacrylic acid stabilized nanoparticle alum (nanoalum) adjuvant governs TH1 differentiation of CD4+ T cells. Nanoscale, 12(4), 2515-2523. DOI: 10.1039/c9nr09936k.

12. Hardin, N. Z., Ravula, T., Di Mauro, G., \& Ramamoorthy, A. (2019). Hydrophobic functionalization of polyacrylic acid as a versatile platform for the development of polymer lipid nanodisks. Small, 15(9), 1-5. DOI: 10.1002/smll.201804813.
13. Huang, L., Wang, J., Huang, S., Siaw-Debrah, F., Nyanzu, M., \& Zhuge, Q. (2019). Polyacrylic acidcoated nanoparticles loaded with recombinant tissue plasminogen activator for the treatment of mice with ischemic stroke. Biochem. Bioph. Res. Co., 516(2), 565-570. https://doi.org/10.1016/j.bbrc.2019.06.079.

14. Elliott, J. E., MacDonald, M., Nie, J., \& Bowman, C. N. (2004). Structure and swelling of poly(acrylic acid) hydrogels: Effect of $\mathrm{pH}$, ionic strength, and dilution on the crosslinked polymer structure. Polymer, 45(5), 1503-1510. DOI: 10.1016/j.polymer.2003.12.040.

15. De la Torre, P. M., Enobakhare, Y., Torrado, G., \& Torrado, S. (2003). Release of amoxicillin from polyionic complexes of chitosan and poly(acrylic acid) study of polymer/polymer and polymer/drug interactions within the network structure. Biomaterials, 24(8), 1499-1506. DOI: 10.1016/S0142-9612(02)00512-43.

16. Hu, Y., Jiang, X., Ding, Y., Ge, H., Yuan, Y., \& Yang, C. (2002). Synthesis and characterization of chitosan-poly (acrylic acid) nanoparticles. Biomaterials, 23(15), 3193-3201. DOI: 10.1016/S01429612(02)00071-6.

17. Ulanski, P., Bothe, E., Hildenbrand, K., Rosiak, J. M., \& von Sonntag, C. (1996). Hydroxyl-radical-induced reactions of poly (acrylic acid); a pulse radiolysis, EPR and product study. Part I. Deoxygenated aqueous solutions. J. Chem. Soc. Perkin Trans. 2, 1, 13-22. DOI: $10.1039 / \mathrm{p} 29960000013$.

18. Ulański, P., Bothe, E., Hildenbrand, K., Rosiak, J. M., \& von Sonntag, C. (1996). Hydroxyl-radical-induced reactions of poly (acrylic acid); a pulse radiolysis, EPR and product study. Part II. Oxygenated aqueous solutions. J. Chem. Soc. Perkin Trans. 2, 1, 23-28. DOI: 10.1039/p29960000023.

19. Chen, B., Jerger, K., Fréchet, J. M. J., \& Szoka, F. C. (2009). The influence of polymer topology on pharmacokinetics: Differences between cyclic and linear PEGylated poly(acrylic acid) comb polymers. J. Control. Release, 140(3), 203-209. DOI: 10.1016/j. jconrel.2009.05.021.

20. Munavirov, B., Gnezdilov, O., Rudakova, M., Antzutkin, O. N., \& Filippov, A. (2013). Interaction of polyacrylic acid with lipid bilayers: Effect of polymer mass. Magn. Reson. Chem., 51(11), 750-755. DOI: 10.1002/mrc.4013.

21. Yessine, M. -A., \& Leroux, J. -C. (2004). Membranedestabilizing polyanions: interaction with lipid bilayers and endosomal escape of biomacromolecules. Adv. Drug. Deliver. Rev., 56(7), 999-1021. DOI: 10.1016/j.addr.2003.10.039.

22. Fujiwara, M., Grubbs, R. H., \& Baldeschwieler, J. D. (1997). Characterization of pH-dependent poly(acrylic acid) complexation with phospholipid vesicles. J. Colloid Interf. Sci., 185(1), 210-216. DOI: 10.1006/jcis.1996.4608

23. Chieng, Y. Y., \& Chen, S. B. (2010). Interaction between poly (acrylic acid) and phospholipid vesicles: Effect of $\mathrm{pH}$, concentration, and molecular weight. J. Phys. Chem. B, 114(14), 4828-4835. DOI:10.1021/ jp1002403.

24. Argentiere, S., Blasi, L., Ciccarella, G., Barbarella, G., Cingolani, R., \& Gigli, G. (2010). Nanogels of poly(acrylic acid): Uptake and release behavior with fluorescent oligothiophene-labeled bovine serum 
albumin. J. Appl. Polym. Sci., 116(5), 2808-2815. DOI: 10.1002/app.31691.

25. Mackiewicz, M., Stojek, Z., \& Karbarz, M. (2019). Synthesis of cross-linked poly(acrylic acid) nanogels in an aqueous environment using precipitation polymerization: unusually high volume change. R. Soc. Open Sci., 6(11), 190981. DOI: 10.1098/ rsos.190981.

26. Rosiak, J. M., \& Ulański, P. (1999). Synthesis of hydrogels by irradiation of polymers in aqueous solution. Radiat. Phys. Chem., 55(2), 139-151. DOI: 10.1016/ S0969-806X(98)00319-3.

27. Ulanski, P., Kadlubowski, S., \& Rosiak, J. M. (2002). Synthesis of poly(acrylic acid) nanogels by preparative pulse radiolysis. Radiat. Phys. Chem., 63(3/6), 533-537. DOI: 10.1016/S0969-806X(01)00549-7.

28. Ghaffarlou, M., Sütekin, S. D., \& Güven, O. (2018). Preparation of nanogels by radiation-induced crosslinking of interpolymer complexes of poly(acrylic acid) with poly(vinyl pyrrolidone) in aqueous medium. Radiat. Phys. Chem., 142, 130-136. DOI: 10.1016/j.radphyschem.2017.04.019.

29. Ghorbaniazar, P., Sepehrianazar, A., Eskandani, M., Nabi-Meibodi, M., Kouhsoltani, M., \& Hamishehkar, H. (2015). Preparation of poly acrylic acid-poly acrylamide composite nanogels by radiation technique. Adv. Pharm. Bull., 5(2), 269-275. DOI: 10.15171/ apb.2015.037.

30. Kadlubowski, S., Ulanski, P., \& Rosiak, J. M. (2012). Synthesis of tailored nanogels by means of two-stage irradiation. Polymer, 53(10), 1985-1991. DOI: 10.1016/j.polymer.2012.03.018.

31. Henke, A., Kadlubowski, S., Ulanski, P., Rosiak, J. M., \& Arndt, K. F. (2005). Radiation-induced crosslinking of polyvinylpyrrolidone-poly(acrylic acid) complexes. Nucl. Instrum. Methods Phys. Res. Sect. B-Beam Interact. Mater. Atoms, 236, 391-398. DOI: 10.1016/j.nimb.2005.04.003.

32. Schmidt, T., Janik, I., Kadłubowski, S., Ulański, P., Rosiak, J. M., Reichelt, R., \& Arndt, K. -F. (2005). Pulsed electron beam irradiation of dilute aqueous poly(vinyl methyl ether) solutions. Polymer, 46(23), 9908-9918. DOI: 10.1016/j.polymer.2005.07.077.

33. Kadlubowski, S., Grobelny, J., Olejniczak, W., Cichomski, M., \& Ulanski, P. (2003). Pulses of fast electrons as a tool to synthesize poly(acrylic acid) nanogels. Intramolecular cross-linking of linear polymer chains in additive-free aqueous solution. Macromolecules, 36(7), 2484-2492. DOI: 10.1021/ ma021628s.
34. Ulanski, P., \& Rosiak, J. M. (1999). The use of radiation technique in the synthesis of polymeric nanogels. Nucl. Instrum. Methods Phys. Res. Sect. B-Beam Interact. Mater. Atoms, 151(1/4), 356-360. DOI: 10.1016/S0168-583X(99)00085-3.

35. Ulański, P., Janik, I., \& Rosiak, J. M. (1998). Radiation formation of polymeric nanogels. Radiat. Phys. Chem., 52(1), 289-294. DOI: 10.1016/S0969806X(98)00155-8.

36. Matusiak, M., Kadlubowski, S., \& Ulanski, P. (2018). Radiation-induced synthesis of poly(acrylic acid) nanogels. Radiat. Phys. Chem., 142, 125-129. DOI: 10.1016/j.radphyschem.2017.01.037.

37. Hoshyar, N., Gray, S., Han, H., \& Bao, G. (2016). The effect of nanoparticle size on in vivo pharmacokinetics and cellular interaction. Nanomedicine, 11(6), 673-692. DOI: 10.2217/nnm.16.5.

38. Mier, W., Babich, J., \& Haberkorn, U. (2014). Is nano too big? Eur. J. Nucl. Med. Mol. Imaging, 41(1), 4-6. DOI: 10.1007/s00259-013-2574-9.

39. Ghimire, A., Zore, O. V., Thilakarathne, V. K., Briand, V. A., Lenehan, P. J., Lei, Y., Kasi, R. M., \& Kumar, C. V. (2015). "Stable-on-the-table" biosensors: hemoglobin-poly(acrylic acid) nanogel bioelectrodes with high thermal stability and enhanced electroactivity. Sensors, 15(9), 23868-23885. DOI: 10.3390/ s150923868.

40. World Health Organization (2018). Stability testing of active and finished pharmaceutical products. WHO Expert Committee Preparations for Pharmaceutical on Specifications, Fifty Second Report, Annex 10. WHO. (Technical Report Series No. 1010).

41. Brasch, U., \& Burchard, W. (1996). Preparation and solution properties of microhydrogels from poly (vinyl alcohol). Macromol. Chem. Phys., 197(1), 223-235. DOI: 10.1002/macp.1996.021970117.

42. Abdelwahed, W., Degobert, G., \& Fessi, H. (2006). A pilot study of freeze drying of poly(epsiloncaprolactone) nanocapsules stabilized by poly(vinyl alcohol): Formulation and process optimization. Int. J. Pharm., 309(1/2), 178-188. DOI: 10.1016/j. ijpharm.2005.10.003.

43. Pikal, M. J. (2004). Mechanisms of protein stabilization during freeze-drying and storage: The relative importance of thermodynamic stabilization and glassy state relaxation dynamics. In J. C. May \& L. Rey (Eds.), Freeze-drying/lyophilization of pharmaceutical and biological products (pp. 63-107). Boca Raton: CRC Press. 